\title{
Neuro-oncology: a selected review of ASCO 2016 abstracts
}

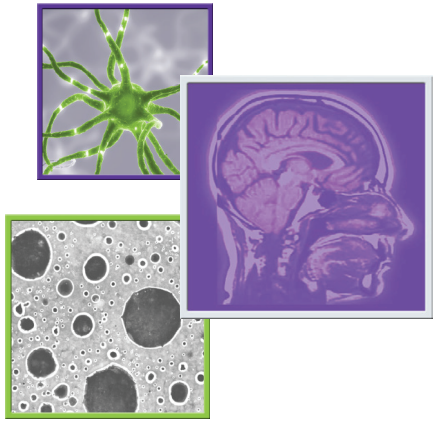

\author{
Marc C Chamberlain*
}

\section{ASCO 2016, 29 May-2 June 2016, Chicago, IL, USA}

The largest annual clinical oncology conference the American Society of Clinical Oncology is held in the USA and gives researchers and other key opinion leaders the opportunity to present new cancer clinical trials and research data. The CNS tumors section of the American Society of Clinical Oncology 2016 covered various aspects of neuro-oncology including metastatic CNS diseases and primary brain tumors, presented via posters, oral talks and over 100 abstracts. This brief review selectively highlights presentations from this meeting in an organizational manner that reflects clinically relevant aspects of a large and multifaceted meeting.

First draft submitted: 16 August 2016 ; Accepted for publication: 18 August 2016; Published online: 12 September 2016

\section{Metastatic disease to the CNS}

- Parenchymal brain metastases

Kayama presented the results of the Japanese JCOG0504 randomized Phase III trial $(\mathrm{n}=271)$ in patients with one to three brain metastases (BMs; any cancer although primarily lung and breast) comparing salvage stereotactic radiosurgery to without whole brain irradiation following initial $\mathrm{BM}$ resection [1]. The median overall survival (OS) was 15.6 months in both arms. Noninferiority of salvage stereotactic radiosurgery versus upfront whole brain irradiation was confirmed by OS.

Kumthekar discussed results of a Phase II trial of ANG1005, a novel brain penetrant taxane derivative, in women with breast cancer and recurrent $\mathrm{BM}$ with or without leptomeningeal metastases [2]. Best intracranial response included $8 / 57(14 \%)$ patients with partial responses (3 [5\%]) and $32 / 57(56 \%)$ with stable disease. For evaluable patients with leptomeningeal metastases, best responses included 5/23 (22\%) partial (2 [9\%] confirmed) and 12/23 (52\%) stable disease. Further evaluation in patients with breast cancer and leptomeningeal metastases is anticipated.

\section{Primary brain tumors}

- Primary CNLS lymphoma

Grommes presented a Phase I trial of ibrutinib in patients with refractory/recurrent primary CNS lymphoma (PCNSL) [3]. Median progression-free survival (PFS) was 6 months. Doses of $840 \mathrm{mg}$ were well tolerated and an expansion cohort is actively enrolling. Molecular correlates of response are ongoing.

*Department of Neurology/Division of Neuro-Oncology, University of Washington, Fred Hutchinson Cancer Research Center, Seattle Cancer Care Alliance, 825 Eastlake Avenue E, POB 19023, MS G4940, Seattle, WA 98109-1023, USA; Tel.: +1 206288 8280;

Fax: +1 206288 2000; marcchamberlain@gmail.com

\section{KEYWORDS}

- ASCO 2016 CNS tumor abstracts $\bullet$ primary and secondary brain tumors 


\section{- Gliomas}

Low-grade gliomas

Upfront trials

Hlavin-Bell presented a mutational analysis of the randomized NRG Oncology/RTOG 9802 Phase III trial in high-risk low-grade gliomas that compared radiotherapy (RT) only to RT + PCV (procarbazine, vincristine, lomustine [CCNU]) chemotherapy [4]. The study examined the prognostic significance of mutations within $I D H 1 / 2$, ATRX, CIC, FUBP1 and the TERT promoter. Observed mutations, by frequency were $74 \%$ $(85 / 115)$ in IDH1/2, TERT promoter in $40 \%$ (32/80), CIC in 25\% (17/69), ATRX in $24 \%$ (16/67) and FUBP1 in 10\% (7/69). In multivariate analysis, TERT promoter (HR: $0.41 ; \mathrm{p}=$ 0.04 ) and $C I C$ mutations (HR: $0.23 ; \mathrm{p}=0.01$ ) were significantly associated with OS, whereas IDH1/2 mutations were significantly associated with PFS (HR: 0.46; p = 0.005). The study emphasizes the prognostic value of TERT promoter, $I D H$ and $C I C$ mutations and suggests that TERT and CIC may provide additional information beyond $I D H$.

\section{Anaplastic gliomas}

\section{Upfront trials}

Van den Bent presented interim results of the Phase III EORTC trial (CATNON) in noncodeleted anaplastic gliomas (AGs) comparing standard RT (RT: 59.4 Gray [Gy] in 30 fractions) to RT + temozolomide (TMZ) [5]. Post-RT there was a second randomization comparing postRT TMZ (up to 12 cycles) versus observation. The analysis compared RT to RT followed by post-RT TMZ. At present there is no comparison assessing the benefit of TMZ given concurrently with RT to RT only. Seven hundred and forty-eight patients were randomized and the primary end point was OS. Interim analysis was prespecified when $40 \%$ of survival events (219 overall) occurred. Hazard ratios favored the post-RT TMZ arm in both PFS (0.586) and OS (0.645). MGMT status could be determined in $74 \%$ of patients and was found methylated in $42 \%$. This practice changing trial suggests RT followed by post-RT TMZ improves OS in noncodeleted AG. Pending are molecular studies addressing the impact of the $I D H$ mutational status and methylation profiling with respect to these recommendations.

Hlavin-Bell examined the prognostic value of mutations within IDH1/2, ATRX, CIC, FUBP1 and the TERT promoter in the prospective randomized Phase III study of anaplastic astrocytoma (AA), RTOG 9814, that compared TMZ to nitrosourea-based primary therapy [6]. Mutations within $I D H 1 / 2$ were found in $55 \%$ (57/104), ATRX in 46\% (28/61), the TERT promoter in $20 \%(11 / 55), C I C$ in $10 \%(6 / 61)$ and FUBP1 in $2 \%(1 / 61)$ of analyzed cases. In multivariate analysis, ATRX mutations were significantly associated with OS (HR: 0.48 ; p $=0.04)$ and PFS (HR: 0.42; p = 0.02), as were IDH1/2 mutations, OS (HR: 0.49, $\mathrm{p}=0.01$ ) and PFS (HR: 0.53, $\mathrm{p}=0.02$ ). The study underscores the prognostic significance of IDH and ATRX, a molecularly defined cohort of AG with improved outcome.

Tabouret presented an analysis of the French POLA glioma network in which 1041 cases of adult diffuse infiltrative gliomas were assessed using the new WHO glioma classification (4th edition) combining molecular and morphologybased pathology [7]. Novel was the finding that AA that are IDH wild-type were divisible into those with $7 \mathrm{p}$ gain and $10 \mathrm{q}$ loss (55\%) and those without these chromosomal number abnormalities. The $7 \mathrm{p}+/ 10 \mathrm{q}$ - subset behaved similar to glioblastoma (GBM) whereas the subset without these abnormalities had improved outcomes suggesting not all AA IDH wild-type should be considered GBM.

Platten presented preliminary data and primarily the study design of a German Phase I trial (NOA-16) of a mutation-specific peptide vaccine targeting newly diagnosed malignant gliomas expressing the IDH1R132H mutation [8]. Eligibility included loss ATRX as well as lacking the $1 \mathrm{p} / 19 \mathrm{q}$ codeletion. The primary end points of the study are safety and immunogenicity as measured by IDH1R132H-specific antibody and T-cell responses. Secondary outcome measures include PFS and overall response rate.

\section{Salvage trials}

Chamberlain reported on the utility of bendamustine, a bifunctional alkylating agent, in 26 patients with TMZ refractory bevacizumab naive AG [9]. Bendamustine appears to have modest single agent activity though the study failed to meet the prespecified criteria of $40 \%$ 6 month PFS.

\section{Glioblastoma}

Upfront trials

Perry presented the NCIC/EORTC Phase III elderly (defined as $<70$ years of age; ECOG 
performance status $0-2)$ GBM trial that compared hypofractionated RT (30 Gy in 15 fractions) to hypofractionated RT plus TMZ followed by post-RT TMZ [10]. Five hundred and eighty-two patients (median age 72 ) were randomized in a 1:1 manner to each treatment arm of which $40 \%$ of 354 analyzable tumors were $M G M T$ methylated. The median number of post-RT TMZ cycles was 5 and $40 \%$ of patients overall received chemotherapy at progression. The study favored the RT + TMZ arm in all three analyzable cohorts wherein median overall OS was 7.6 versus 9.3 months, median OS in the MGMT methylated cohort was 7.7 versus 13.5 months and median OS in the unmethylated cohort was 3.7 versus 5.3 months. Similar advantages were seen with respect to PFS. This practice changing study concluded all elderly patients are best treated by hypofractionated RT + TMZ irrespective of the $M G M T$ promoter methylation status however this biomarker has significant prognostic and predictive value (doubling OS) although not affecting treatment allocation. Unlike the Nordic and German NOA-08 elderly GBM trials, there was no TMZ only treatment arm that had previously been recommended for MGMT methylated tumors. Controversy will continue however as there is no study that compares standard versus hypofractionated RT + TMZ whereupon it remains likely elderly patients with GBM and good performance will continue to receive therapy that varies by provider and institutional philosophy [11].

Nguyen explored the relationship between $M G M T$ methylation and $h T E R T$ promoter mutation in 303 IDH1 wild-type primary GBM [12]. Human telomerase reverse transcriptase ( $h T E R T$ ) promoter mutation, has been shown to enhance the expression of telomerase and occurs in $70-80 \%$ of GBM. There is currently no consensus regarding the clinical significance of the mutation of $h T E R T$ in GBM. According to Nguyen et al. although hTERT promoter mutation alone was not prognostic of GBM outcome, it strongly interacted with $M G M T$ promoter methylation and appeared to enable the survival benefit from $M G M T$ promoter methylation.

When examining the prognostic role of EGFRvIII in 109 EGFR-amplified GBM patients, Weller et al. found that EGFRvIII was not prognostic in these patients and that at recurrence, the majority of EDFRvIII-positive
GBM maintains positivity [13]. Weller explained that at recurrence, expression of EGFRvIII could be lost or newly gained in small subsets of patients. Therefore, re-biopsy with reassessment of EGFRvIII status is recommended for recurrent GBM patients enrolled in clinical trials using EGFRvIII-targeting agents.

\section{Salvage trials}

Desjardins presented early data (15 patients) from a dose-escalation trial of the oncolytic polio/rhinovirus recombinant (PVSRIPO) in patients with recurrent GBM [14]. PVSRIPO recognizes CD155, an oncofetal cell adhesion molecule and tumor antigen widely expressed ectopically in malignancy. PVSRIPO was delivered intratumorally by convection-enhanced delivery. A median OS of 12.6 months was observed for the PVSRIPO group versus 10.5 months for an institutional historical control groups, with $23.3 \%$ of PVSRIPO patients alive at 24 months versus $13.7 \%$ of historical controls. Study accrual continues in conjunction with dose escalation of PVSRIPO.

Holdhoff assessed interinterpreter reliability of neuropathology following re-resection of recurrent GBM following primary treatment with RT + TMZ. Their findings (providing case samples to 44 neuropathologists across 30 centers) demonstrated marginal interinterpreter reliability in pathological assessment of disease status in re-resected GBM [15]. The authors conclude more formal and consistent criteria (none are currently found in the WHO criteria) are urgently needed as inconsistent assessment of disease status directly impacts on patient selection for clinical trials, as well as on decision making in clinical practice.

Wick presented results of the Phase III EORTC study (26101) that compared CCNU to bevacizumab + CCNU [16]. OS was 9.1 versus 8.6 months and PFS 4.2 versus 1.5 months in the combination arm versus the CCNU arm, respectively. This study is practice changing and has resulted in CCNU pre-empting bevacizumab as the nontrial agent of first choice for recurrent GBM.

Groves presented a small $(\mathrm{n}=21)$ Phase II study of the prodrug aldodoxorubicin in patients with first recurrent GBM [17]. Aldodoxorubicin is a prodrug of doxorubicin that is attached to a $\mathrm{pH}$ sensitive linker that binds covalently to albumin and is released preferentially within tumors. Three partial responses and seven stable patterns 
were seen suggesting efficacy in recurrent GBM however no PFS data were provided.

Mau-Sorensen reviewed a Phase II study using the novel brain-penetrant agent selinexor, an antagonist of the nuclear export protein exportin [18]. Selinexor forces the nuclear retention and activation of tumor suppressor proteins such as $p 53, I k B$ and $p 21$. In a subset of patients, prerepeat resection treatment demonstrated intratumoral concentrations exceeding the $\mathrm{IC}_{50}$ established in vitro. RANO defined best response (investigator determined) among 27 evaluable patients included three partial responses (11\%), six stable $(22 \%)$ and 18 progressive disease $(67 \%)$ in first recurrent bevacizumab naive GBM. The 6 month-PFS rate is immature, although four patients continued on therapy at 6 months. Accrual to study continues.

A small Phase II trial of TRC 105, antiendoglin monoclonal antibody, in combination with bevacizumab was presented by Ahluwalia. He suggested that there was improved activity with this combination relative to single agent bevacizumab [19]. The endothelial cell membrane receptor endoglin, is essential for angiogenesis and is highly expressed on tumor vessels. Endoglin is upregulated by hypoxia and VEGF inhibition; it could be an important mechanism of VEGF resistance. A randomized Phase II trial in bevacizumab naive GBM patients is currently evaluating treatment with the TRC105/bevacizumab combination.

Elinzano discussed the use of a monoclonal antibody-drug conjugate, prostate-specific membrane antigen-antibody drug conjugate (PSMA ADC), directed against the prostate specific membrane antigen that is upregulated in GBM [20]. PSMA ADC however had no demonstrable activity in patients with progressive GBM likely due to minimal expression of the PSMA target and was associated with dose-limiting toxicity.

Reardon outlined a multiarm clinical trial using the anti-PD-1 monoclonal antibody, durvalumab (MEDI3736), in both the recurrent and upfront setting of GBM that is currently enrolling.

Brenner presented data from the Phase II trial of ofranogene obadenovec, VB-111, a gene therapy specifically targeting angiogenic endothelial cells administered in combination with bevacizumab [21]. Forty-six patients received VB-111 as a single agent given bimonthly and upon progression, 24 received VB-111 with BEV. VB-111 was safe and well-tolerated alone and combined with BEV in recurrent GBM. VB-111 continuous exposure significantly increased OS compared with a historical BEV meta-analysis controls. A randomized Phase III trial is currently enrolling in recurrent GBM.

Multiple Phase I trials were presented including the use of a brain penetrating pan-proteasome inhibitor, marizomib in conjunction with bevacizumab; a brain-penetrant PI3K/mTOR inhibitor, GDC-0084; $\mathrm{T}$ cells redirected to EGFRvIII with a chimeric antigen receptor; dianhydrogalactitol (VAL-083), a bi-functional alkylating agent that readily crosses the bloodbrain barrier; intratumoral viral delivery of the adenovirus vector, Ad-RTS-IL-12 and the activator ligand veledimex; TPI287, a brain penetrable third-generation taxane used in combination with bevacizumab; MLN0128 an oral TORC1/2 inhibitor in combination with bevacizumab; plerixafor, a CXCR4 antagonist (the CXCL12/CXCR4 axis is instrumental in radiation induced tumor vasculature recovery), given in combination with RT + TMZ; AZD 1775, a first in class Weel inhibitor; carboxyamidotriazole orotate (an oral inhibitor of nonvoltage dependent calcium signaling) and RT with concurrent and adjuvant TMZ; pembrolizumab in combination with bevacizumab; nivolumab with or without bevacizumab; and S49076, an oral multitarget inhibitor of $c-M E T, A X L$, FGFR in combination with bevacizumab [22-35].

Prados discussed a feasibility trial wherein tumor in patients with recurrent GBM underwent whole exome and RNA sequencing from which targeted therapy recommendations were made on average in less than 4 weeks [36]. Efficacy studies are planned using this strategy.

\section{Conclusion}

Several practice changing trials were presented this year at ASCO most notably the use of hypofractionated RT + TMZ in elderly patients with GBM regardless of tumor MGMT methylation (CCTG CE.6, EORTC 26062-22061, TROG 08.02, NCT00482677), upfront RT + TMZ in non-1p/19q codeleted AG (CATNON) and $\mathrm{CCNU}$ for first recurrent GBM in patients not otherwise considered for a clinical trial (EORTC 26101). Molecular profiling of tumors from two Phase III randomized clinical trials (RTOG 9802 and RTOG 9813) illustrate new subsets of intermediate grade gliomas of prognostic significance that likely will impact design of future 
trials. At last, a multitude of Phase I trials in GBM indicate the range of therapeutics being investigated in GBM with an increasing number of therapies utilizing cancer immunotherapy, currently a major focus of clinical trials in high-grade gliomas that hopefully will influence clinical trial design and clinical practice.

\section{Disclosure}

The author collected and analyzed data.

\section{Financial \& competing interests disclosure}

The author has no relevant affliations or financial involvement with any organization or entity with a financial interest in or financial conflict with the subject matter or materials discussed in the manuscript. This includes employment, consultancies, honoraria, stock ownership or options, expert testimony, grants or patents received or pending, or royalties.

No writing assistance was utilized in the production of this manuscript.

\section{References}

1 Kayama T, Sato S, Sakurado K et al. JCOG0504: a Phase III randomized trial of surgery with whole brain radiation therapy versus surgery with salvage stereotactic radiosurgery in patients with 1 to 4 brain metastases. J. Clin. Oncol. 33(15s), Abstract 2003 (2015).

2 Kumathekar P, Tang SC, Brenner AJ et al. ANG1005, a novel brain-penetrant taxane derivative, for the treatment of recurrent brain metastases and leptomeningeal carcinomatosis from breast cancer. J. Clin. Oncol. 33(15s), Abstract 2004 (2015).

3 Grommes C, Kaley TJ, Nolan C et al. Phase I study of single agent ibrutinib in recurrent/ refractory primary (PCNSL) and secondary CNS lymphoma (SCNSL). J. Clin. Oncol. 33(15s), Abstract 2046 (2015).

4 Hlavin-Bell E, McElroy JP, Fleming J et al. Comprehensive mutational analysis in NRG Oncology/RTOG 9802: A Phase III trial of RT vs RT + PCV in high-risk low grade gliomas (LGG). J. Clin. Oncol. 34(18s), Abstract 2017 (2016).

5 Van den Bent MJ, Erridge S, Vogelbaum MA et al. Results of the interim analysis of the EORTC randomized Phase III CATNON trial on concurrent and adjuvant temozolomide in anaplastic glioma without $1 \mathrm{p} / 19 \mathrm{q}$ co-deletion: an intergroup trial. J. Clin. Oncol. 34(18s), Abstract LBA2000 (2016).

6 Hlavin-Bell E, McElroy JP, Fleming J et al. Comprehensive mutational analysis in NRG Oncology/RTOG 9813: a Phase III trial of $\mathrm{RT}+\mathrm{TMZ}$ vs RT + nu for anaplastic astrocytoma and mixed anaplastic oligoastrocytoma (Astrocytoma Dominant). J. Clin. Oncol. 34(18s), Abstract 2016 (2016).

7 Tabouret E, Nguyen AT, Dehais C et al. Validation of the new glioma WHO classification in the French POLA network: analysis of 1041 cases. J. Clin. Oncol. 34(18s), Abstract 2015 (2016).
8 Platten M, Schilling D, Bunse T et al. A mutation-specific peptide vaccine targeting IDH1R132H in patients with newly diagnosed malignant astrocytomas: a first-in-man multicenter Phase 1 clinical trial of the German Neurooncology Working Group (NOA-16) with newly diagnosed malignant astrocytomas: a first-in-man multicenter Phase I clinical trial of the German Neurooncology Working Group (NOA-16). J. Clin. Oncol. 34(18s), Abstract TPS2082 (2016).

9 Chamberlain MC, Colman H, Raizer JJ. Bendamustine for anaplastic gliomas. J. Clin. Oncol. 33(15s), Abstract 2051 (2015).

10 Perry PR, Laperriere N, O'Callaghan CJ et al. A Phase III randomized controlled trial of short-course radiotherapy with or without concomitant and adjuvant temozolomide in elderly patients with glioblastoma (CCTG CE.6, EORTC 26062-22061, TROG 08.02, NCT00482677). J. Clin. Oncol. 34(18s), Abstract LBA2 (2016).

11 Huang J, Parker Sampson P, Mabry Perkins S et al. Trends in utilization and impact of concurrent chemotherapy with radiation therapy for elderly patients with glioblastoma: A review of the National Cancer Data Base. J. Clin. Oncol. 34(18s), Abstract 2034 (2016).

12 Nguyen HN, Li T, Lie A et al. Benefit of $M G M T$ methylation in glioblastoma in relation to hTERT promoter mutation. J. Clin. Oncol. 34(18s), Abstract 2006 (2016).

13 Weller M, Hentschel B, Kaulich K et al. EGFR gene amplification and variant III (EGFRvIII) mutation in primary and recurrent glioblastoma. J. Clin. Oncol. 34(18s), Abstract 2042 (2016).

14 Desjardins A, Sampson JH, Peters KB et al. Patient survival on the dose escalation phase of the Oncolytic Polio/Rhinovirus Recombinant (PVSRIPO) against WHO grade IV malignant glioma (MG) clinical trial compared to historical controls. J. Clin. Oncol. 33(15s), Abstract 2061 (2015).
15 Holdhoff M, Piotrowski AF, Ye X et al. Inter-interpreter reliability of neuropathological assessment of disease status after early repeat resection for suspected recurrent glioblastoma. J. Clin. Oncol. 34(18s), Abstract 2050 (2016).

16 Wick W, Stupp R, Gorlia T et al. EORTC 26101 Phase III trial exploring the combination of bevacizumab and lomustine in patients with first progression of a glioblastoma. J. Clin. Oncol. 33(15s), Abstract 2001 (2015).

17 Groves MD, Portnow J, Boulmay BC et al. Phase 2 study of aldodoxorubicin in relapsed glioblastoma. J. Clin. Oncol. 34(18s), Abstract 2027 (2016).

18 Mau-Sorensen M, Plotkin SR, Wen PY et al. A Phase 2 study on efficacy, safety and intratumoral pharmacokinetics of oral selinexor (KPT-330) in patients with recurrent glioblastoma (GBM). J. Clin. Oncol. 34(18s), Abstract 2077 (2016).

19 Ahluwalia MS, Rogers LR, Chaudhary RT et al. A Phase 2 trial of TRC105 with bevacizumab for bevacizumab refractory glioblastoma. J. Clin. Oncol. 33(15s), Abstract 2035 (2015).

20 Elinzano H, Hebda N, Luppe D et al. PSMA ADC for progressive glioblastoma: Phase II Brown University Oncology Research Group Study. J. Clin. Oncol. 34(18s), Abstract 2065 (2016).

21 Brenner AJ, Cohen YC, Vredenburgh JJ et al. Ofranogene obadenovec (VB-111), an anti-cancer gene therapy in combination with bevacizumab to improve overall survival compared to bevacizumab monotherapy in patients with rGBM: a Phase 2 historically controlled trial. J. Clin. Oncol. 33(15s), Abstract 2074 (2015).

22 Bota DA, Desjardins A, Mason WP et al. Phase 1, multicenter, open-label, doseescalation, study of marizomib (MRZ) and bevacizumab (BEV) in WHO grade IV malignant glioma (G4 MG). J. Clin. Oncol. 34(18s), Abstract 2037 (2016). 
23 Wen PY, Cloughesy TF, Olivero A et al. A first-in-human Phase 1 study to evaluate the brain-penetrant $\mathrm{PI} 3 \mathrm{~K} / \mathrm{mTOR}$ inhibitor GDC-0084 in patients with progressive or recurrent high-grade glioma. J. Clin. Oncol. 34(18s), Abstract 2012 (2016).

24 O'Rourke DM, Nasrallah M, Morrissette JJ et al. Pilot study of T-cells redirected to EGFRvIII with a chimeric receptor in patients with EGFRvIII+ glioblastoma. J. Clin. Oncol. 34(18s), Abstract 2067 (2016).

25 Shih KC, Patel MR, Butowski NA et al. Phase I/II study of VAL-083 in patients with recurrent glioblastoma. J. Clin. Oncol. 34(18s), Abstract 2063 (2016).

26 Lebel FM, Barrett JA, Chiocca EA et al. Effect of controlled intratumoral viral delivery of Ad-RTS-hIL-12 + oral veledimex in subjects with recurrent or progressive glioma. J. Clin. Oncol. 34(18s), Abstract 2052 (2016).

27 Goldlust SA, Nabors LB, Mohile N et al. Phase $1 / 2$ trial of bevacizumab plus TPI 287, a brain penetrable anti-microtubule agent, in patients with recurrent glioblastoma. J. Clin. Oncol. 34(18s), Abstract 2055 (2016).
28 Nayak L, Hays JL, Muzikansky A et al. A Phase I study of MLN0128 and bevacizumab in patients with recurrent glioblastoma and other solid tumors. J. Clin. Oncol. 33(15s), Abstract 2013 (2015).

29 Thomas RP, Nagpal S, Iv M et al. A Phase I study of chemo-radiotherapy with plerixafor for newly diagnosed glioblastoma (GB). J. Clin. Oncol. 33(15s), Abstract 2068 (2015).

30 Sanai N, Boerner J, Dhruv H et al. Phase 0 trial of AZD1775 in patients with firstrecurrence glioblastoma. J. Clin. Oncol. 33(15s), Abstract 2008 (2015).

31 Omura AMP, Beal K, McNeill KA et al. Phase IB trial of carboxyamidotriazole orotate (CTO) and radiotherapy (RT) with concurrent and adjuvant temozolomide (TMZ) in newly diagnosed glioblastoma (GBM). J. Clin. Oncol. 33(15s), Abstract 2060 (2015).

32 Reardon DA, De Groot JF, Colman $\mathrm{H}$ et al. Safety of pembrolizumab in combination with bevacizumab in recurrent glioblastoma (rGBM). J. Clin. Oncol. 33(15s), Abstract 2010 (2015).
33 Reardon DA, Sampson JH, Sahebjam S et al. Safety and activity of nivolumab (nivo) monotherapy and nivo in combination with ipilimumab (ipi) in recurrent glioblastoma (GBM): Updated results from checkmate-143. J. Clin. Oncol.33(15s), Abstract 2014 (2015)

34 Reardon DA, Kaley TJ, Dietrich J et al. Phase 2 study to evaluate the clinical efficacy and safety of MEDI4736 (durvalumab) in patients with glioblastoma (GBM). J. Clin. Oncol. 34(18s), Abstract TPS2080 (2016).

35 Hoang-Xuan K, Hottinger A, Royer-Perron L et al. Phase I/II study of S49076, a multitarget inhibitor of c-MET, AXL, FGFR in combination with bevacizumab in patients with recurrent glioblastoma. J. Clin. Oncol. 33(15s), Abstract 2033 (2015).

36 Prados M, Kuhn JG, Colman H et al. Precision medicine in recurrent glioblastoma: a feasibility trial conducted by the Ivy Foundation Early Phase Clinical Trials Consortium. J. Clin. Oncol. 33(15s), Abstract 2031 (2015). 